\title{
Schottky Diode Derating for Survivability in a Heavy Ion Environment
}

\author{
Megan C. Casey ${ }^{1}$, Jean-Marie Lauenstein ${ }^{1}$, \\ Raymond L. Ladbury ${ }^{1}$, Edward P. Wilcox ${ }^{2}$, Alyson D. Topper ${ }^{2}$, \\ Anthony Phan², Hak Kim², and Kenneth A. LaBel ${ }^{1}$
}




\section{List of Acronyms and Symbols}

- SEE - Single Event Effects

- NEPP - NASA Electronic Parts and Packaging Program

- $\mathrm{V}_{\mathrm{R}}$ - Reverse Voltage

- $V_{F}$ - Forward Voltage

- $I_{R}$ - Reverse Current

- $I_{F}$ - Forward Current

- LBNL - Lawrence Berkeley National Laboratory
- DUT - Device Under Test

- LET - Linear Energy Transfer

- MOSFET - Metal Oxide Semiconductor Field Effect Transistor

- $\phi_{B}-$ Schottky Barrier Height 


\section{Introduction}

- In 2011/2012, Bob Gigliuto of GSFC observed failures in the output Schottky diodes of DC/DC converters

- Independent testing of the diodes was undertaken to determine their vulnerability to heavy ions

- Until this point, diodes generally were not considered to be susceptible to SEEs

- These diode failures could be catastrophic to scientific instruments, or even entire spacecraft

- Power MOSFETs are derated when operating in radiation environments

- Would a similar approach work for Schottky diodes? 


\section{Test Facilities and Technique}

- All parts were tested at Lawrence Berkeley National Laboratory's (LBNL) 88" cyclotron with $1233 \mathrm{MeV}$ Xe (LET $=58.8 \mathrm{MeV}-\mathrm{cm}^{2} / \mathrm{mg}$ )

- All diodes were reverse biased while irradiated

- After each beam run, $V_{F}, V_{R}$, and

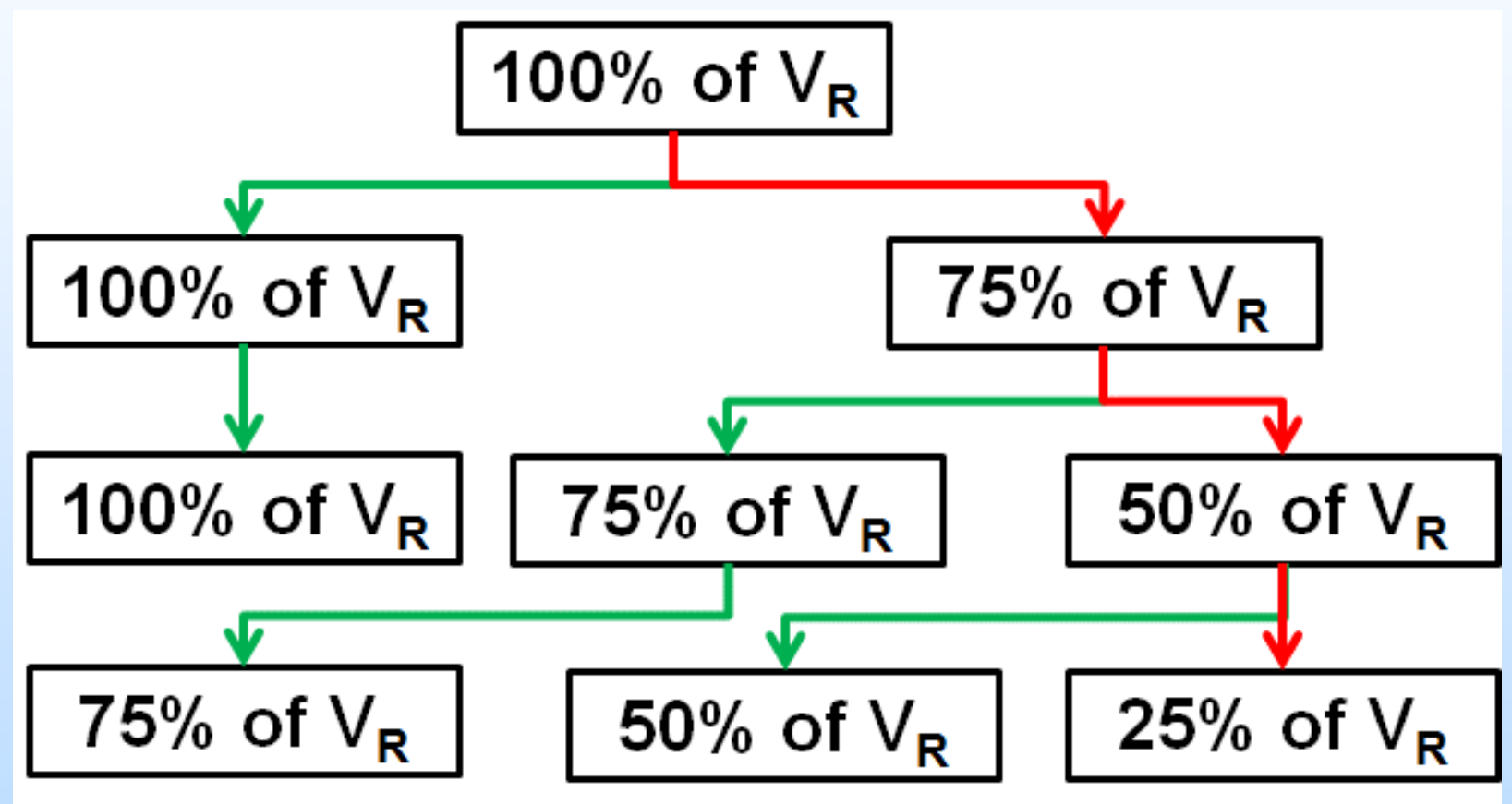
$I_{R}$ were measured 


\section{Parts Tested}

- 38 Schottky diodes from 8 manufacturers (See the paper for details.)

- Reverse voltages range from $40 \mathrm{~V}$ to $200 \mathrm{~V}$

- Forward currents (per diode) from $5 \mathrm{~A}$ to $30 \mathrm{~A}$

- Within the manufacturers, high temperature, high forward voltage lines are compared to low temperature, low forward voltage and low barrier height lines 


\section{Observed Radiation Responses}
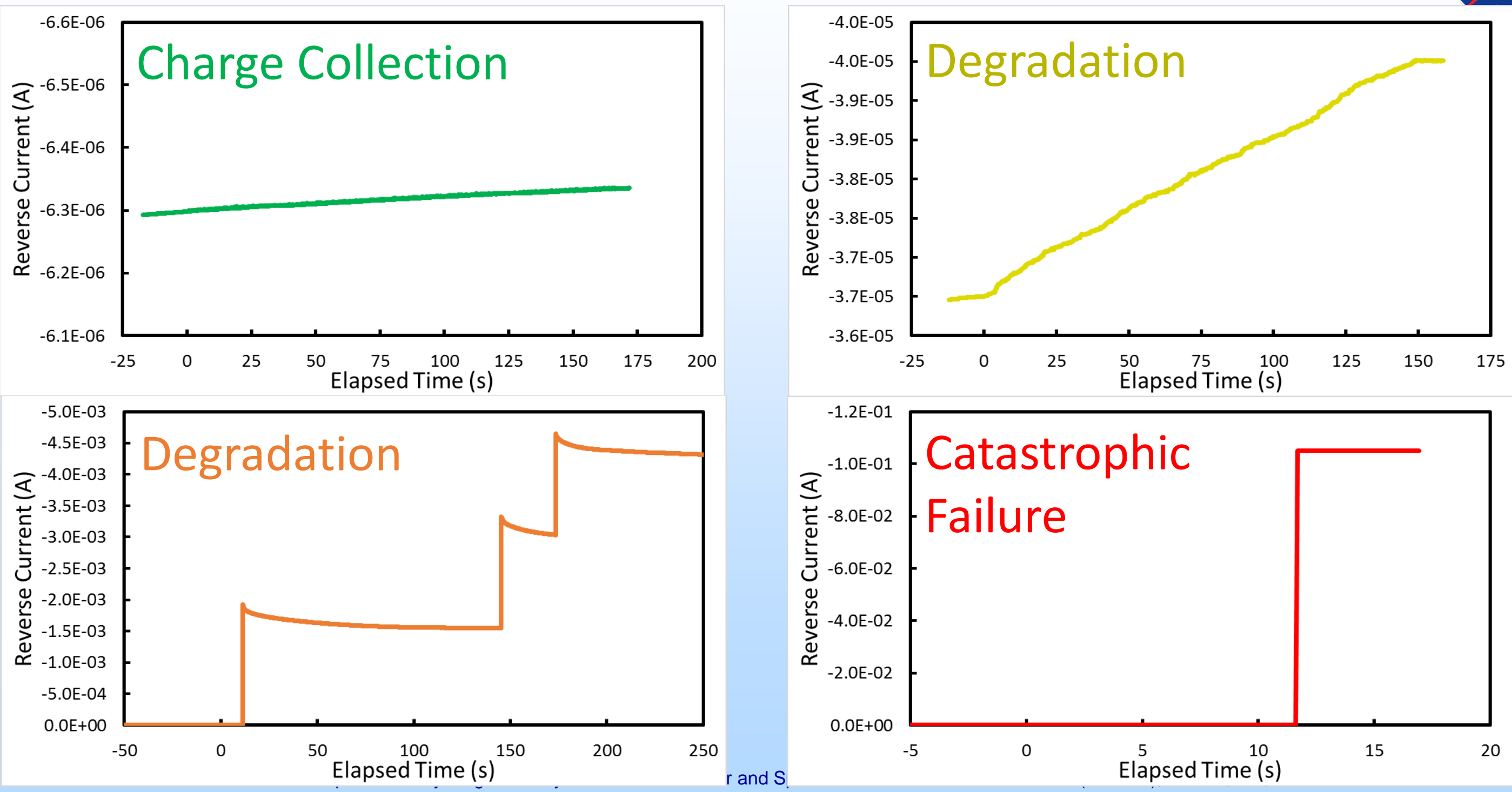


\section{Results}

$\mathbb{Q}$ Not Tested $\square$ Pass $\quad \square$ Degradation and Pass $\quad \square$ Degradation and Failure $\square$ Catastrophic Failure $100 \%$

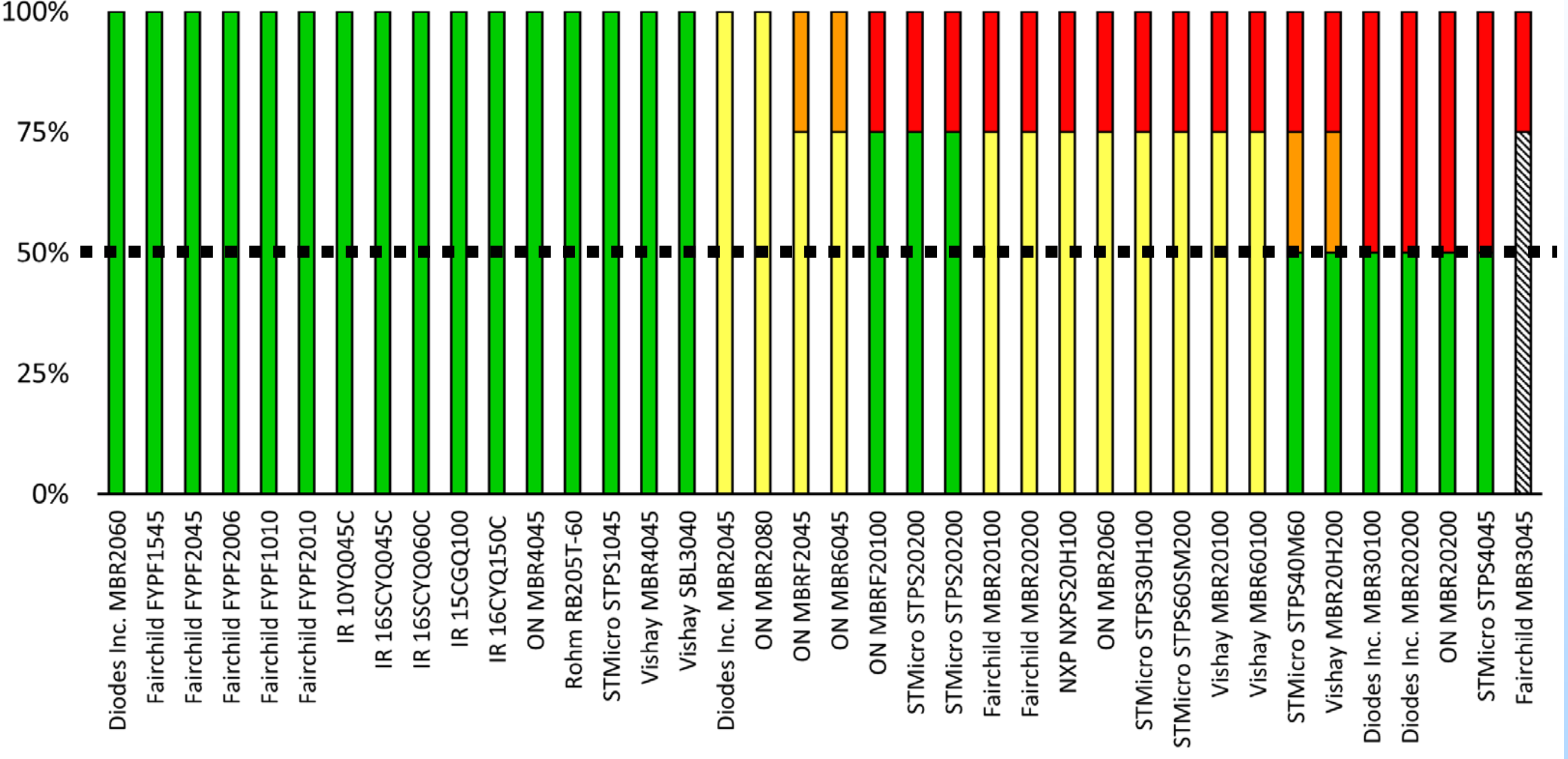




\section{Results}

$100 \%$ of Reverse Voltage

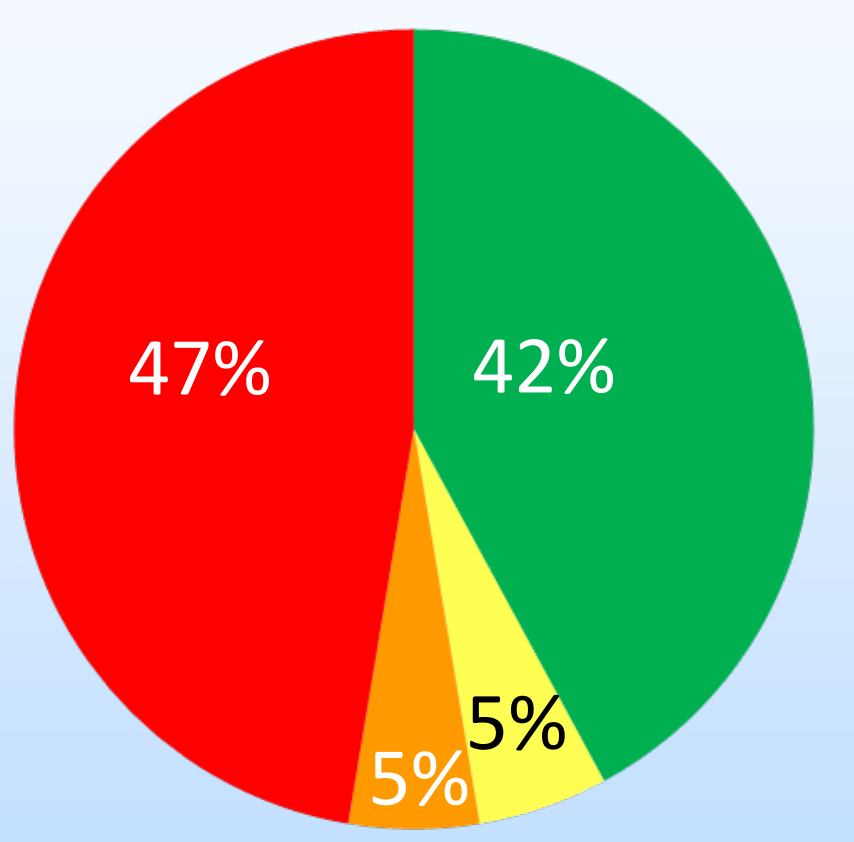

$75 \%$ of Reverse Voltage

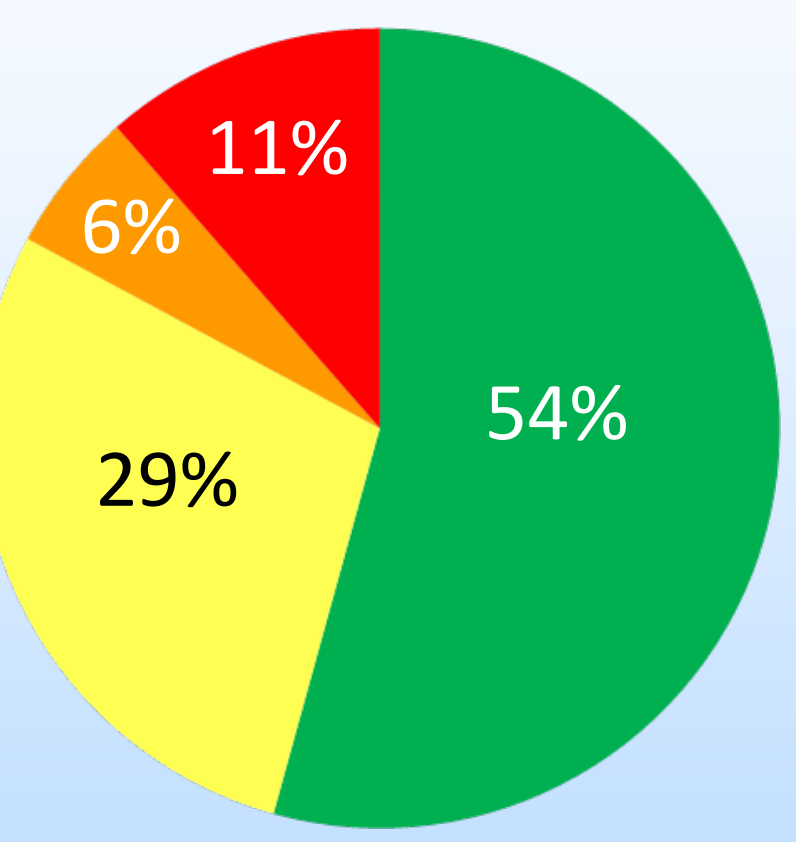

$50 \%$ of Reverse Voltage

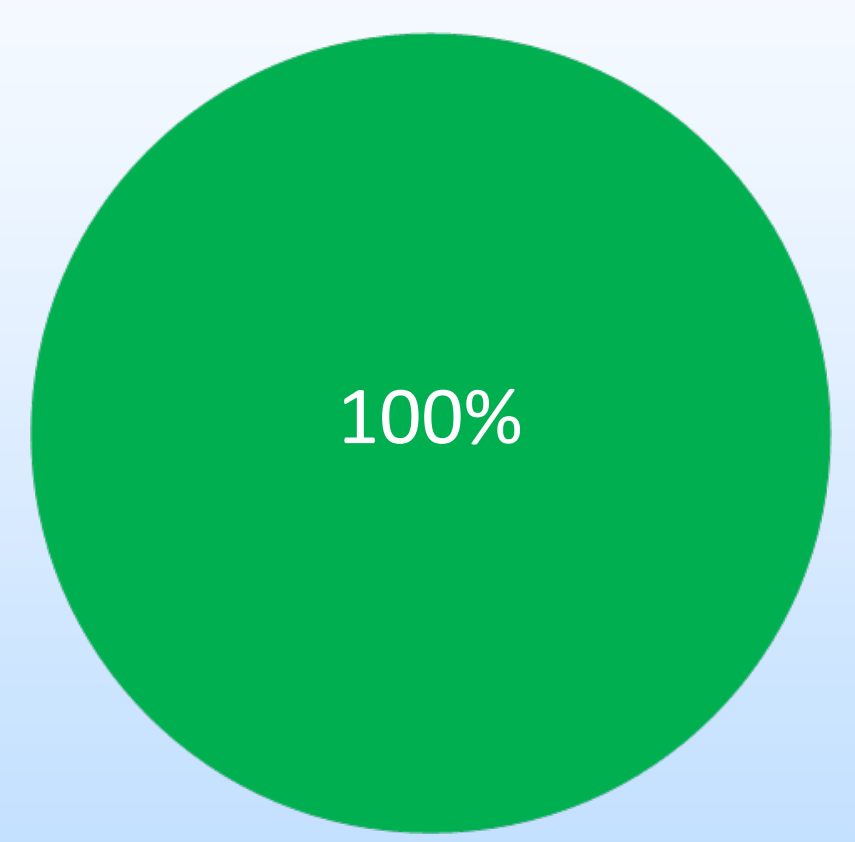

By derating to $50 \%$ of the reverse voltage, all failures are eliminated for the parts tested 


\section{Failures as a Function of Barrier Height}
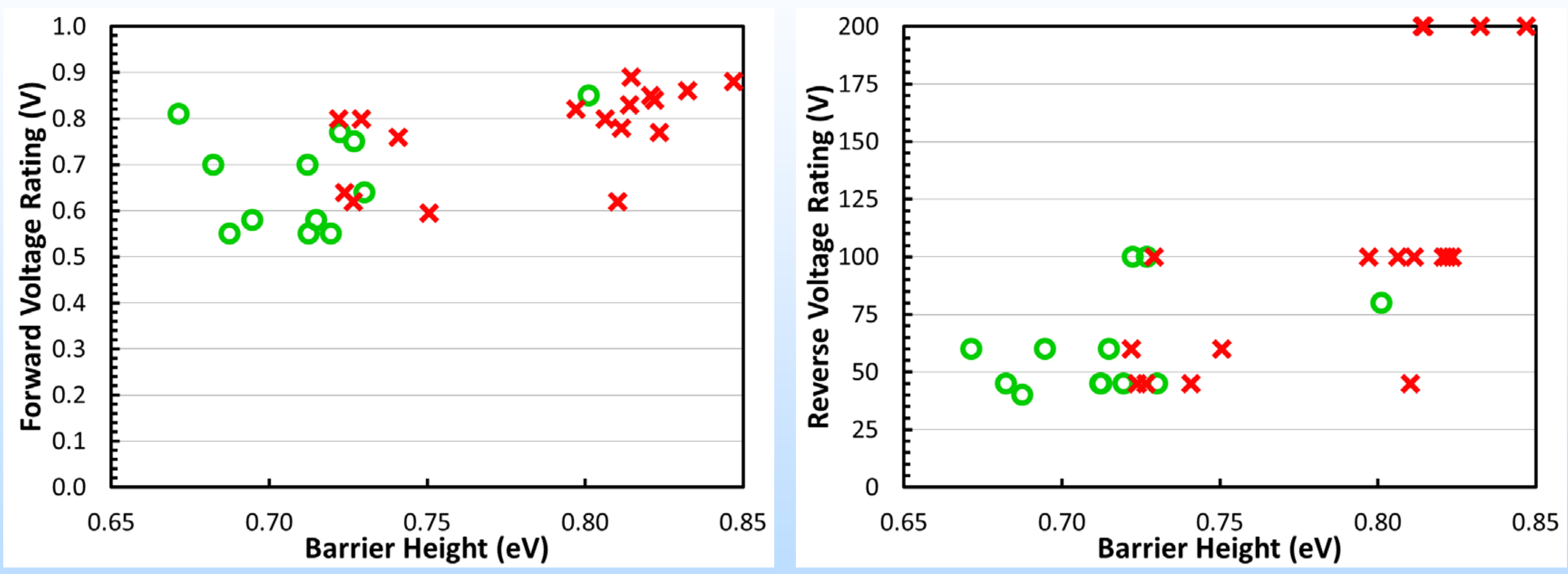

No failures observed in parts with $\phi B$ less than $0.72 \mathrm{eV}$ 


\section{Failures as a Function of Reverse Current}

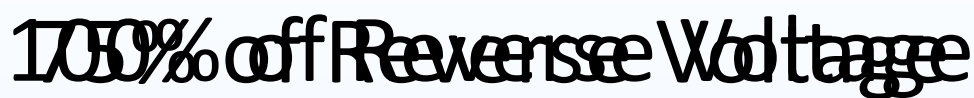
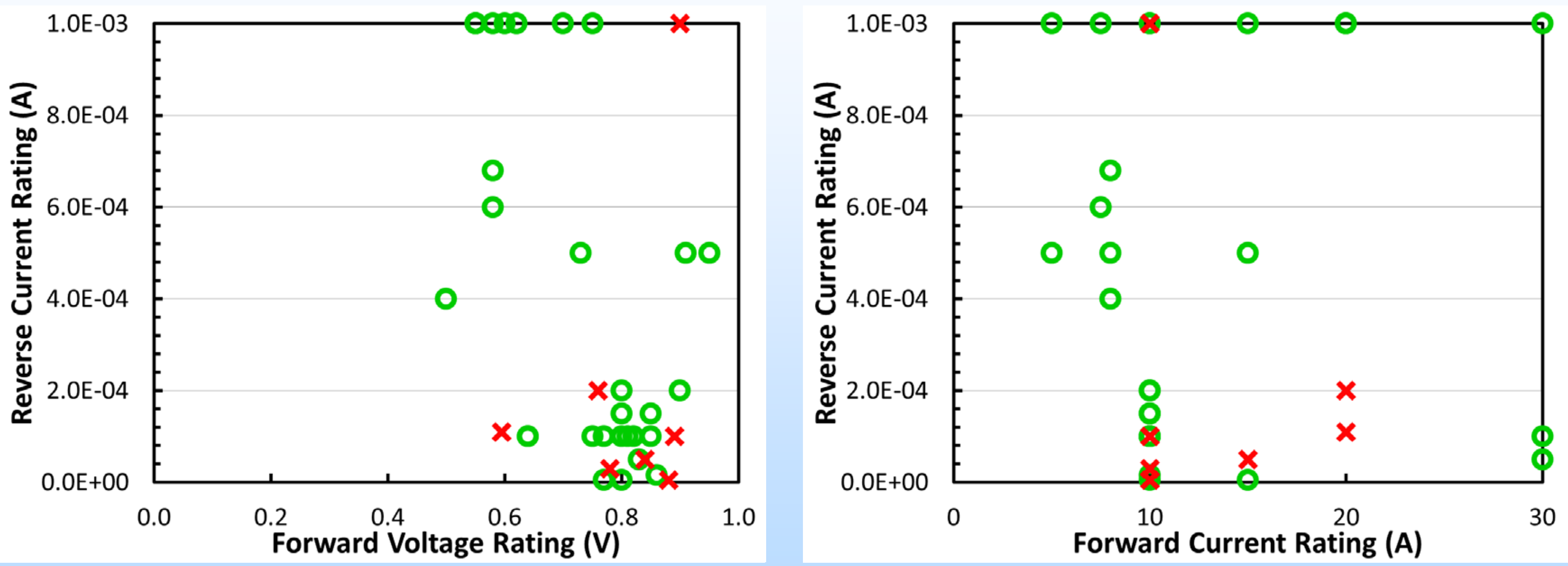

Strong correlation in susceptibility and low $I_{R}$ rating 


\section{Thermal Imaging of Failed Schottky Diodes}
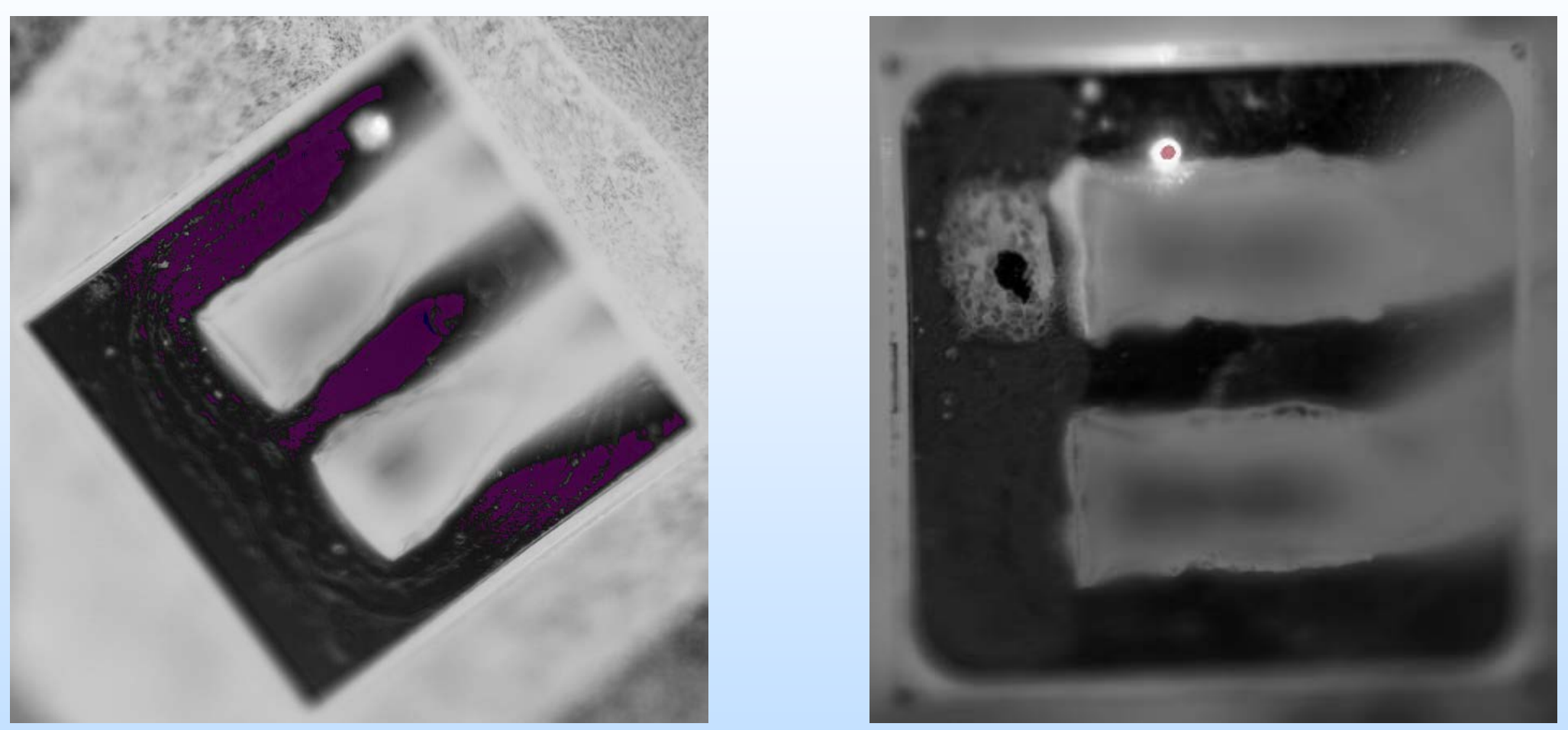

Failure locations are hundreds of microns away from guardring 


\section{Conclusions}

- Schottky diodes are susceptible to destructive SEEs

- Failures only occur when diodes are reverse biased

- Thermal images show failures are not located solely along guardring

- Failures are much more widespread than originally suspected

- Failures observed across manufacturers, reverse voltages, and forward current ratings tested

- No failures observed at $50 \%$ (or below) of rated reverse voltage

- There appears to be a strong correlation between failures and barrier height, as well as reverse current rating

- SEE testing should be considered when selecting parts with $\phi_{\mathrm{B}}>0.72 \mathrm{eV}$ or with $\mathrm{I}_{\mathrm{R}} \leq 200 \mu \mathrm{A}$

- Correlation also exists between failures and forward voltage 


\section{Acknowledgments}

- This work was sponsored by the NASA Electronic Parts and Packaging Program.

- The authors gratefully acknowledge members of the Radiation Effects and Analysis Group who contributed to the test results presented here.

- The authors would also like to acknowledge the staff at the LBNL cyclotron for their support during these tests. 


\section{Parts Tested}

\begin{tabular}{|l|l|c|c|c|c|}
\hline Part Number & Manufacturer & $\begin{array}{c}\text { Reverse } \\
\text { Voltage (V) }\end{array}$ & $\begin{array}{c}\text { Forward } \\
\text { Current (A) }\end{array}$ & $\begin{array}{c}\text { Reverse } \\
\text { Current (A) }\end{array}$ & $\begin{array}{c}\text { Forward } \\
\text { Voltage (V) }\end{array}$ \\
\hline MBR2045 & Diodes Inc. & 45 & 10 & $1.00 \mathrm{E}-04$ & 0.64 \\
\hline MBR2060 & Diodes Inc. & 60 & 10 & $1.00 \mathrm{E}-04$ & 0.81 \\
\hline MBRF30100 & Diodes Inc. & 100 & 15 & $5.00 \mathrm{E}-05$ & 0.84 \\
\hline MBR20200 & Diodes Inc. & 200 & 10 & $1.00 \mathrm{E}-04$ & 0.89 \\
\hline FYPF1545 & Fairchild Semiconductor & 45 & 7.5 & $1.00 \mathrm{E}-03$ & 0.55 \\
\hline FYPF2045 & Fairchild Semiconductor & 45 & 10 & $1.00 \mathrm{E}-03$ & 0.55 \\
\hline FYPF2006 & Fairchild Semiconductor & 60 & 10 & $1.00 \mathrm{E}-03$ & 0.58 \\
\hline FYPF1010 & Fairchild Semiconductor & 100 & 5 & $1.00 \mathrm{E}-03$ & 0.75 \\
\hline FYPF2010 & Fairchild Semiconductor & 100 & 10 & $1.00 \mathrm{E}-04$ & 0.77 \\
\hline MBR3045 & Fairchild Semiconductor & 45 & 15 & $1.00 \mathrm{E}-03$ & 0.62 \\
\hline MBR20100 & Fairchild Semiconductor & 100 & 10 & $2.00 \mathrm{E}-04$ & 0.8 \\
\hline MBR20200 & Fairchild Semiconductor & 200 & 10 & $2.00 \mathrm{E}-04$ & 0.9 \\
\hline 10YQ045C & International Rectifier & 45 & 5 & $5.00 \mathrm{E}-04$ & 0.73 \\
\hline $16 S C Y Q 045 C$ & International Rectifier & 45 & 8 & $4.00 \mathrm{E}-04$ & 0.5 \\
\hline $16 S C Y Q 060 C$ & International Rectifier & 60 & 8 & $6.80 \mathrm{E}-04$ & 0.58 \\
\hline 15 CGQ100 & International Rectifier & 100 & 15 & $5.00 \mathrm{E}-04$ & 0.95 \\
\hline $16 C Y Q 150 C$ & International Rectifier & 150 & 8 & $5.00 \mathrm{E}-04$ & 0.91 \\
\hline NXPS20H100 & NXP Semiconductor & 100 & 10 & $4.50 \mathrm{E}-06$ & 0.77 \\
\hline RB205T-60 & Rohm Semiconductor & 60 & 7.5 & $6.00 \mathrm{E}-04$ & 0.58 \\
\hline
\end{tabular}

\begin{tabular}{|l|l|c|c|c|c|}
\hline Part Number & Manufacturer & $\begin{array}{c}\text { Reverse } \\
\text { Voltage (V) }\end{array}$ & $\begin{array}{c}\text { Forward } \\
\text { Current (A) }\end{array}$ & $\begin{array}{c}\text { Reverse } \\
\text { Current (A) }\end{array}$ & $\begin{array}{c}\text { Forward } \\
\text { Voltage (V) }\end{array}$ \\
\hline MBRF2045 & ON Semiconductor & 45 & 10 & $1.00 \mathrm{E}-04$ & 0.64 \\
\hline MBR4045 & ON Semiconductor & 45 & 20 & $1.00 \mathrm{E}-03$ & 0.7 \\
\hline MBR6045 & ON Semiconductor & 45 & 30 & $1.00 \mathrm{E}-03$ & 0.62 \\
\hline MBR2060 & ON Semiconductor & 60 & 10 & $1.50 \mathrm{E}-04$ & 0.8 \\
\hline MBR2080 & ON Semiconductor & 80 & 10 & $1.00 \mathrm{E}-04$ & 0.85 \\
\hline MBRF20100 & ON Semiconductor & 100 & 10 & $1.50 \mathrm{E}-04$ & 0.85 \\
\hline MBR20200 & ON Semiconductor & 200 & 10 & $1.00 \mathrm{E}-03$ & 0.9 \\
\hline STPS1045 & STMicroelectronics & 45 & 10 & $1.00 \mathrm{E}-04$ & 0.75 \\
\hline STPS4045 & STMicroelectronics & 45 & 20 & $2.00 \mathrm{E}-04$ & 0.76 \\
\hline STPS40M60 & STMicroelectronics & 60 & 20 & $1.10 \mathrm{E}-04$ & 0.595 \\
\hline STPS20100 & STMicroelectronics & 100 & 10 & $3.00 \mathrm{E}-05$ & 0.78 \\
\hline STPS30H100 & STMicroelectronics & 100 & 15 & $5.00 \mathrm{E}-06$ & 0.8 \\
\hline STPS20200 & STMicroelectronics & 200 & 10 & $1.50 \mathrm{E}-05$ & 0.86 \\
\hline STPS60SM200 & STMicroelectronics & 200 & 30 & $5.00 \mathrm{E}-05$ & 0.83 \\
\hline MBR4045 & Vishay Semiconductor & 45 & 20 & $1.00 \mathrm{E}-03$ & 0.6 \\
\hline MBR20100 & Vishay Semiconductor & 100 & 10 & $1.00 \mathrm{E}-04$ & 0.8 \\
\hline MBR60100 & Vishay Semiconductor & 100 & 30 & $1.00 \mathrm{E}-04$ & 0.82 \\
\hline MBR20H200 & Vishay Semiconductor & 200 & 10 & $5.00 \mathrm{E}-06$ & 0.88 \\
\hline SBL3040 & Vishay Semiconductor & 40 & 15 & $1.00 \mathrm{E}-03$ & 0.55 \\
\hline
\end{tabular}




\section{Results}

\begin{tabular}{|c|c|c|c|c|c|c|c|c|}
\hline Part Number & Manufacturer & $\begin{array}{c}\text { Reverse } \\
\text { Voltage (V) }\end{array}$ & $\begin{array}{c}\text { Forward } \\
\text { Current }(\mathrm{A})\end{array}$ & $\begin{array}{c}\text { Reverse } \\
\text { Current }(A)\end{array}$ & $\begin{array}{c}\text { Forward } \\
\text { Voltage (V) }\end{array}$ & $100 \%$ & $75 \%$ & $50 \%$ \\
\hline MBR2045 & Diodes Inc. & 45 & 10 & $1.00 \mathrm{E}-04$ & 0.64 & D & NT & NT \\
\hline MBR2060 & Diodes Inc. & 60 & 10 & $1.00 \mathrm{E}-04$ & 0.81 & $\mathbf{P}$ & NT & NT \\
\hline MBRF30100 & Diodes Inc. & 100 & 15 & $5.00 \mathrm{E}-05$ & 0.84 & CF & $\mathrm{CF}$ & $\mathbf{P}$ \\
\hline MBR20200 & Diodes Inc. & 200 & 10 & $1.00 E-04$ & 0.89 & $\mathrm{CF}$ & CF & $\mathbf{P}$ \\
\hline FYPF1545 & Fairchild Semiconductor & 45 & 7.5 & $1.00 \mathrm{E}-03$ & 0.55 & $\mathbf{P}$ & NT & NT \\
\hline FYPF2045 & Fairchild Semiconductor & 45 & 10 & $1.00 E-03$ & 0.55 & $\mathbf{P}$ & NT & NT \\
\hline FYPF2006 & Fairchild Semiconductor & 60 & 10 & $1.00 \mathrm{E}-03$ & 0.58 & $\mathbf{P}$ & NT & NT \\
\hline FYPF1010 & Fairchild Semiconductor & 100 & 5 & $1.00 \mathrm{E}-03$ & 0.75 & $\mathbf{P}$ & NT & NT \\
\hline FYPF2010 & Fairchild Semiconductor & 100 & 10 & $1.00 \mathrm{E}-04$ & 0.77 & $\mathbf{P}$ & NT & NT \\
\hline MBR3045 & Fairchild Semiconductor & 45 & 15 & $1.00 \mathrm{E}-03$ & 0.62 & CF & NT & NT \\
\hline MBR20100 & Fairchild Semiconductor & 100 & 10 & $2.00 \mathrm{E}-04$ & 0.8 & CF & D & NT \\
\hline MBR20200 & Fairchild Semiconductor & 200 & 10 & $2.00 \mathrm{E}-04$ & 0.9 & CF & $\mathrm{D}$ & NT \\
\hline 10YQ045C & International Rectifier & 45 & 5 & $5.00 \mathrm{E}-04$ & 0.73 & $\mathbf{p}$ & NT & NT \\
\hline $16 \mathrm{SCYQ} 045 \mathrm{C}$ & International Rectifier & 45 & 8 & 4.00E-04 & 0.5 & $\mathbf{p}$ & NT & NT \\
\hline 16SCYQ060C & International Rectifier & 60 & 8 & $6.80 \mathrm{E}-04$ & 0.58 & $\mathbf{p}$ & NT & NT \\
\hline 15CGQ100 & International Rectifier & 100 & 15 & $5.00 \mathrm{E}-04$ & 0.95 & $\mathbf{p}$ & NT & NT \\
\hline 16CYQ150C & International Rectifier & 150 & 8 & $5.00 \mathrm{E}-04$ & 0.91 & $\mathrm{p}$ & NT & NT \\
\hline NXPS20H100 & NXP Semiconductor & 100 & 10 & $4.50 \mathrm{E}-06$ & 0.77 & CF & $\mathrm{D}$ & NT \\
\hline RB205T-60 & Rohm Semiconductor & 60 & 7.5 & $6.00 \mathrm{E}-04$ & 0.58 & $\mathrm{p}$ & NT & NT \\
\hline
\end{tabular}

\begin{tabular}{|l|l|c|c|c|c|c|c|c|}
\hline Part Number & Manufacturer & $\begin{array}{c}\text { Reverse } \\
\text { Voltage (V) }\end{array}$ & $\begin{array}{c}\text { Forward } \\
\text { Current (A) }\end{array}$ & $\begin{array}{c}\text { Reverse } \\
\text { Current (A) }\end{array}$ & $\begin{array}{c}\text { Forward } \\
\text { Voltage (V) }\end{array}$ & $\mathbf{1 0 0 \%}$ & $\mathbf{7 5 \%}$ & $\mathbf{5 0 \%}$ \\
\hline MBRF2045 & ON Semiconductor & 45 & 10 & $1.00 \mathrm{E}-04$ & 0.64 & DF & D & NT \\
\hline MBR4045 & ON Semiconductor & 45 & 20 & $1.00 \mathrm{E}-03$ & 0.7 & P & NT & NT \\
\hline MBR6045 & ON Semiconductor & 45 & 30 & $1.00 \mathrm{E}-03$ & 0.62 & DF & D & NT \\
\hline MBR2060 & ON Semiconductor & 60 & 10 & $1.50 \mathrm{E}-04$ & 0.8 & CF & D & NT \\
\hline MBR2080 & ON Semiconductor & 80 & 10 & $1.00 \mathrm{E}-04$ & 0.85 & D & NT & NT \\
\hline MBRF20100 & ON Semiconductor & 100 & 10 & $1.50 \mathrm{E}-04$ & 0.85 & CF & P & NT \\
\hline MBR20200 & ON Semiconductor & 200 & 10 & $1.00 \mathrm{E}-03$ & 0.9 & CF & CF & P \\
\hline STPS1045 & STMicroelectronics & 45 & 10 & $1.00 \mathrm{E}-04$ & 0.75 & P & NT & NT \\
\hline STPS4045 & STMicroelectronics & 45 & 20 & $2.00 \mathrm{E}-04$ & 0.76 & CF & CF & P \\
\hline STPS40M60 & STMicroelectronics & 60 & 20 & $1.10 \mathrm{E}-04$ & 0.595 & CF & CF & P \\
\hline STPS20100 & STMicroelectronics & 100 & 10 & $3.00 \mathrm{E}-05$ & 0.78 & CF & CF & P \\
\hline STPS30H100 & STMicroelectronics & 100 & 15 & $5.00 \mathrm{E}-06$ & 0.8 & CF & D & NT \\
\hline STPS20200 & STMicroelectronics & 200 & 10 & $1.50 \mathrm{E}-05$ & 0.86 & CF & P & NT \\
\hline STPS60SM200 & STMicroelectronics & 200 & 30 & $5.00 \mathrm{E}-05$ & 0.83 & CF & D & NT \\
\hline MBR4045 & Vishay Semiconductor & 45 & 20 & $1.00 \mathrm{E}-03$ & 0.6 & P & NT & NT \\
\hline MBR20100 & Vishay Semiconductor & 100 & 10 & $1.00 \mathrm{E}-04$ & 0.8 & CF & D & NT \\
\hline MBR60100 & Vishay Semiconductor & 100 & 30 & $1.00 \mathrm{E}-04$ & 0.82 & CF & D & NT \\
\hline MBR20H200 & Vishay Semiconductor & 200 & 10 & $5.00 \mathrm{E}-06$ & 0.88 & CF & DF & P \\
\hline SBL3040 & Vishay Semiconductor & 40 & 15 & $1.00 \mathrm{E}-03$ & 0.55 & P & NT & NT \\
\hline
\end{tabular}

Catastrophic Failure

Degradation and Failure

Degradation and Pass

Pass

Not Tested 


\section{Failures as a Function of Forward Voltage}
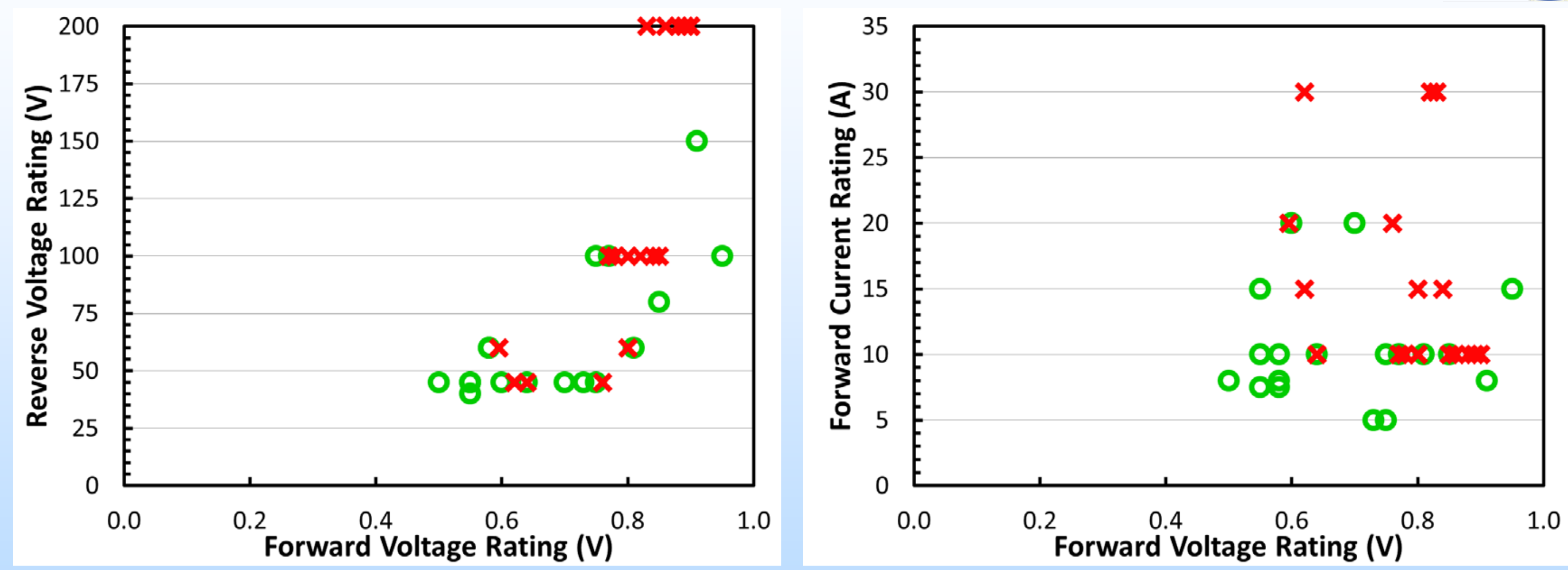

Weaker correlation in susceptibility and high $\mathrm{V}_{\mathrm{F}}$ rating However, product lines billed as low $V_{F}$ or low $\phi_{B}$ show very little susceptibility 